\title{
INVERSE EIGENVALUE PROBLEM FOR EUCLIDEAN DISTANCE MATRICES OF SIZE 3
}

\author{
GAŠPER JAKLIČ and JOLANDA MODIC ${ }^{凶}$
}

(Received 31 January 2012; accepted 18 July 2012)

\begin{abstract}
A matrix is a Euclidean distance matrix (EDM) if there exist points such that the matrix elements are squares of distances between the corresponding points. The inverse eigenvalue problem (IEP) is as follows: construct (or prove the existence of) a matrix with particular properties and a given spectrum. It is well known that the IEP for EDMs of size 3 has a solution. In this paper all solutions of the problem are given and their relation with geometry is studied. A possible extension to larger EDMs is tackled.
\end{abstract}

2010 Mathematics subject classification: primary 15A18; secondary 15A29.

Keywords and phrases: Euclidean distance matrix, inverse eigenvalue problem, bordered matrix.

\section{Introduction}

A matrix $D \in \mathbb{R}^{n \times n}$ is a Euclidean distance matrix (EDM) if there exist points $\boldsymbol{x}_{i} \in \mathbb{R}^{r}$, $i=1,2, \ldots, n$, such that $d_{i j}=\left\|\boldsymbol{x}_{i}-\boldsymbol{x}_{j}\right\|^{2}$. The minimal $r$ is called an embedding dimension. EDMs were introduced by Menger in 1928, and later studied by Schoenberg [11] and other authors. They have many interesting properties, and are used in various applications in linear algebra, graph theory and bioinformatics, for example, where frequently the question arises of what can be said about a configuration of points $\boldsymbol{x}_{i}$ if only distances between them are known.

By their construction EDMs are symmetric, hollow (with zeros on the diagonal) and nonnegative. A nonzero EDM has only one positive eigenvalue and the sum of its eigenvalues is zero. More properties can be found in $[4,8,9]$. There are several nice characterisations of EDMs, see [8], for example. We will need the following result.

Theorem 1.1 [8]. Let $D \in \mathbb{R}^{n \times n}$ be a nonzero symmetric hollow matrix. The matrix $D$ is an EDM if and only if it has only one positive eigenvalue and there exists $\boldsymbol{w} \in \mathbb{R}^{n}$ such that $D \boldsymbol{w}=\boldsymbol{e}$ and $\boldsymbol{w}^{T} \boldsymbol{e} \geq 0$. Here $\boldsymbol{e}:=\left[\begin{array}{llll}1 & 1 & \ldots\end{array}\right]^{T} \in \mathbb{R}^{n}$.

This research was funded in part by the European Union, European Social Fund, Operational Programme for Human Resources, Development for the Period 2007-2013.

(C) 2012 Australian Mathematical Publishing Association Inc. 0004-9727/2012 \$16.00 
An inverse eigenvalue problem (IEP) is as follows. Given numbers $\lambda_{1}, \lambda_{2}, \ldots, \lambda_{n} \in$ $\mathbb{C}$, is it possible to construct (or prove the existence of) a matrix $D$, satisfying some prescribed requirements, such that $\lambda_{i}$ are the eigenvalues of $D$ ? Such problems are encountered in various applications, for example in civil engineering when studying vibrations of buildings: the frequencies (eigenvalues) are prescribed, and one needs to construct an appropriate matrix.

IEPs are hard when additional requirements are given, such as $d_{i j} \geq 0, i, j=$ $1,2, \ldots, n, D=D^{T}$, and $d_{i i}=0, i=1,2, \ldots, n$ (see [10], for example). The problem for such a setup was solved by Fiedler (see [6, Theorem 2.2]). More on IEPs in general can be found in the nice monograph [3], and for some particular problems see $[2,5,10]$.

The IEP for EDMs is as follows: for given real numbers $\lambda_{1}>0 \geq \lambda_{2} \geq \cdots \geq \lambda_{n}$ such that $\sum_{i} \lambda_{i}=0$, construct an $\operatorname{EDM} D$ which has the spectrum $\left\{\lambda_{1}, \lambda_{2}, \ldots, \lambda_{n}\right\}$. For $n=2$, the solution is simply

$$
\left[\begin{array}{cc}
0 & -\lambda_{2} \\
-\lambda_{2} & 0
\end{array}\right]
$$

For $n=3$, it turns out that Fiedler's solution (see [6]) yields an EDM

$$
\left[\begin{array}{ccc}
0 & -\lambda_{2} & \sqrt{\frac{\left(\lambda_{2}+\lambda_{3}\right) \lambda_{3}}{2}} \\
\sqrt{\frac{-\lambda_{2}}{\frac{\left(\lambda_{2}+\lambda_{3}\right) \lambda_{3}}{2}}} & \sqrt{\frac{\left(\lambda_{2}+\lambda_{3}\right) \lambda_{3}}{2}} & \sqrt{\frac{\left(\lambda_{2}+\lambda_{3}\right) \lambda_{3}}{2}} \\
0
\end{array}\right]
$$

since it corresponds to points

$$
(0,0), \quad\left(\sqrt{\frac{\lambda_{2}}{4}+\sqrt{\frac{\left(\lambda_{2}+\lambda_{3}\right) \lambda_{3}}{2}}}, \pm \frac{\sqrt{-\lambda_{2}}}{2}\right),
$$

that form an isosceles triangle (see [8]). This is a cornerstone for the construction of a solution of the IEP for some larger $n$ [8]. The smallest open problem is $n=7$, and the construction for larger $n$ is related to the well-known problem of the existence of Hadamard matrices.

Fiedler's construction, based on bordered matrices, was analysed in [8]. It was shown that it has considerable limitations for large $n$.

A natural question arises whether Fiedler's solution for $n=3$ is unique. If not, some other solution could perhaps have some advantage in solving the IEP for EDMs.

In this paper we show that there are infinitely many solutions of the cubic IEP for EDMs. A nice interplay between analysis, geometry and linear algebra can be seen. A relation between eigenvalues of a hollow symmetric matrix and its bordered matrix suggests that such a construction could be used to find a solution of the IEP for EDMs for some larger $n$. The paper is concluded by studying the step from the quadratic to the cubic case and some remarks on problems encountered for larger $n$. 


\section{Inverse eigenvalue problem for the cubic case}

Take $\lambda_{1}>0 \geq \lambda_{2} \geq \lambda_{3}$, such that $\lambda_{1}+\lambda_{2}+\lambda_{3}=0$. Without loss of generality we can assume that the points $\boldsymbol{x}_{i}$, corresponding to the EDM $D$ that we are looking for, are $(0,0),(a, 0),(b, c)$, where $a>0, b>0, c>0$. This follows from invariance of EDMs for rotations and reflections. Thus

$$
D=\left[\begin{array}{ccc}
0 & a^{2} & b^{2}+c^{2} \\
a^{2} & 0 & (a-b)^{2}+c^{2} \\
b^{2}+c^{2} & (a-b)^{2}+c^{2} & 0
\end{array}\right]
$$

Its characteristic polynomial is

$$
\begin{aligned}
p(x)=-x^{3} & +2 x\left(\left(a^{2}-a b+b^{2}\right)^{2}+\left((a-b)^{2}+b^{2}\right) c^{2}+c^{4}\right) \\
+ & 2 a^{2}\left((a-b)^{2}+c^{2}\right)\left(b^{2}+c^{2}\right) .
\end{aligned}
$$

Note that the quadratic term is missing, and the coefficients at the linear term and the constant are positive. Descartes' rule of signs reveals that there are two negative eigenvalues and one positive. Since the polynomial $p$ is a depressed cubic, it it easy to obtain solutions by Cardano's method.

On the other hand,

$$
p(x)=-\left(x-\lambda_{1}\right)\left(x-\lambda_{2}\right)\left(x-\lambda_{3}\right) .
$$

A comparison of coefficients gives the nonlinear system

$$
\begin{gathered}
2 a^{2}\left((a-b)^{2}+c^{2}\right)\left(b^{2}+c^{2}\right)=-\lambda_{2} \lambda_{3}\left(\lambda_{2}+\lambda_{3}\right), \\
2\left(\left(a^{2}-a b+b^{2}\right)^{2}+\left((a-b)^{2}+b^{2}\right) c^{2}+c^{4}\right)=\lambda_{2}^{2}+\lambda_{2} \lambda_{3}+\lambda_{3}^{2} .
\end{gathered}
$$

A brief look at the geometry of the problem reveals that there are 24 solutions: there are triangles with base length equal to $a, \sqrt{b^{2}+c^{2}}, \sqrt{(b-a)^{2}+c^{2}}$ representing a rotation or reflection of the original triangle. For each of them, the $y$-flip $b \leftrightarrow(a-b)$ gives two possibilities. Thus there are six solutions, but if we consider the possibilities $\pm a, \pm b, \pm c$ there are four in each case, which altogether gives 24 solutions.

Thus, if $a$ is an admissible solution, then the choices $a=\sqrt{b^{2}+c^{2}}$ and $a=$ $\sqrt{(b-a)^{2}+c^{2}}$ also give admissible solutions that yield the same triangle, just in a different position.

By solving the system (2.1) for unknowns $b$ and $c$, we obtain an appropriate solution

$$
\begin{aligned}
& b=\frac{a^{5}+\sqrt{-a^{4}\left(a^{2}+\lambda_{1}\right)\left(a^{2}+\lambda_{2}\right)\left(a^{2}+\lambda_{3}\right)}}{2 a^{4}} \\
& c=\frac{1}{2 a^{2}} \sqrt{2 a^{3} \sqrt{-\left(a^{2}-\lambda_{1}\right)\left(a^{2}-\lambda_{2}\right)\left(a^{2}-\lambda_{3}\right)}+\lambda_{1} \lambda_{2} \lambda_{3}-a^{2}\left(\lambda_{2}^{2}+\lambda_{2} \lambda_{3}+\lambda_{3}^{2}\right)} .
\end{aligned}
$$


For the solution at hand,

$$
\begin{aligned}
d_{12} & =d_{12}(a):=a^{2} \\
d_{13} & =d_{13}(a):=b^{2}+c^{2} \\
& =\frac{\sqrt{-\left(a^{2}-\lambda_{1}\right)\left(a^{2}-\lambda_{2}\right)\left(a^{2}-\lambda_{3}\right)}-\sqrt{-\left(a^{2}+\lambda_{1}\right)\left(a^{2}+\lambda_{2}\right)\left(a^{2}+\lambda_{3}\right)}}{2 a}, \\
d_{23} & =d_{23}(a):=(b-a)^{2}+c^{2} \\
& =\frac{\sqrt{-\left(a^{2}-\lambda_{1}\right)\left(a^{2}-\lambda_{2}\right)\left(a^{2}-\lambda_{3}\right)}+\sqrt{-\left(a^{2}+\lambda_{1}\right)\left(a^{2}+\lambda_{2}\right)\left(a^{2}+\lambda_{3}\right)}}{2 a} .
\end{aligned}
$$

The requirement $d_{13} \geq 0$ yields $2 \lambda_{1} \lambda_{2} \lambda_{3} \geq 0$. The last expression is clearly positive. This gives the following result.

Theorem 2.1. The matrix

$$
D=\left[\begin{array}{ccc}
0 & d_{12} & d_{13} \\
d_{12} & 0 & d_{23} \\
d_{13} & d_{23} & 0
\end{array}\right],
$$

defined by (2.3), is nonnegative, hollow, and has prescribed eigenvalues $\lambda_{1}>0 \geq \lambda_{2} \geq$ $\lambda_{3}$ with $\lambda_{1}+\lambda_{2}+\lambda_{3}=0$ if and only if its elements are well defined, that is,

$$
-\lambda_{2} \leq a^{2} \leq-\lambda_{3}
$$

Let us denote $I:=\left[\sqrt{-\lambda_{2}}, \sqrt{-\lambda_{3}}\right]$. In order for $D$ to be an EDM, we need additional requirements-the points $\boldsymbol{x}_{1}=(0,0), \boldsymbol{x}_{2}=(a, 0), \boldsymbol{x}_{3}=(b, c)$ have to exist. Thus we are looking for parameters $a \in I$ such that

$$
\begin{aligned}
f(a):=2 a^{3} \sqrt{-\left(a^{2}+\lambda_{2}+\lambda_{3}\right)\left(a^{2}-\lambda_{2}\right)\left(a^{2}-\lambda_{3}\right)} \\
-\left(\lambda_{2}+\lambda_{3}\right) \lambda_{2} \lambda_{3}-a^{2}\left(\lambda_{2}^{2}+\lambda_{2} \lambda_{3}+\lambda_{3}^{2}\right) \geq 0 .
\end{aligned}
$$

There is at least one admissible $a$ since the choice $a=\sqrt{-\lambda_{2}}$ yields the well-known solution (1.1) (with a triangle in a different position). This follows from

$$
f\left(\sqrt{-\lambda_{2}}\right)=\lambda_{2}^{2}\left(\lambda_{2}+2 \sqrt{2} \sqrt{\lambda_{3}\left(\lambda_{2}+\lambda_{3}\right)}\right) \geq \lambda_{2}^{2}\left(\lambda_{2}+2 \sqrt{2} \sqrt{2 \lambda_{2}^{2}}\right)=-3 \lambda_{2}^{3} \geq 0 .
$$

A brief analysis of

$$
f\left(\sqrt{-\lambda_{3}}\right)=\lambda_{3}^{2}\left(\lambda_{3}+2 \sqrt{2} \sqrt{\lambda_{2}\left(\lambda_{2}+\lambda_{3}\right)}\right)
$$

reveals that it is nonnegative for $\lambda_{3} \in\left[2(2+\sqrt{6}) \lambda_{2}, \lambda_{2}\right]$. For such a $\lambda_{3}$ each $a \in I$ leads to a solution of the IEP for EDMs.

If $\lambda_{3}<2(2+\sqrt{6}) \lambda_{2}$, the analysis is more complex. For $\lambda_{3}=2(2+\sqrt{6}) \lambda_{2}$, $f\left(\sqrt{-\lambda_{3}}\right)=0$, but there is another interesting zero,

$$
a_{z}:=\frac{\sqrt{-\lambda_{3}}}{2}=\sqrt{1+\sqrt{\frac{3}{2}}} \sqrt{-\lambda_{2}},
$$



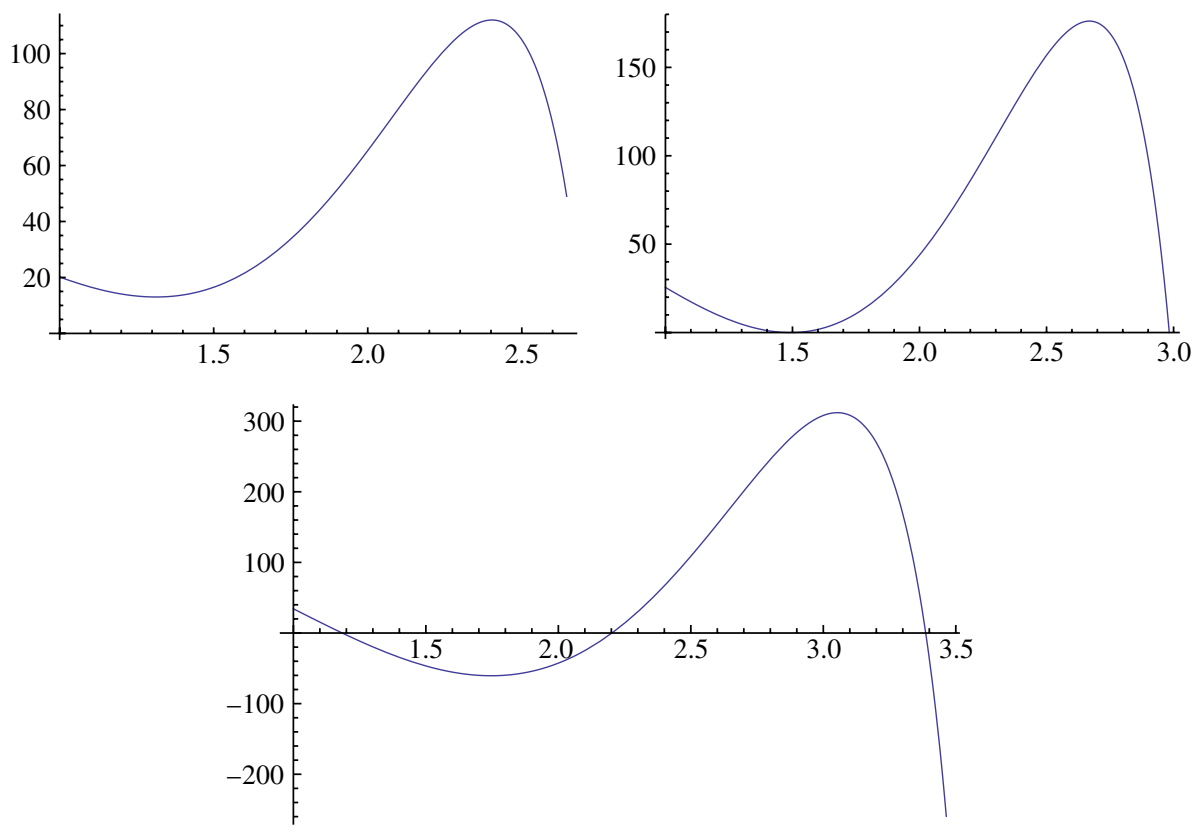

FIGURE 1. Shape of the function $f$ for small $t:=\lambda_{3} / \lambda_{2}$ (top left), $t=2(2+\sqrt{6})$ (top right), and for large $t$ (bottom) on interval $I$. Here $\lambda_{2}=-1$.

in $I$ which is also a local minimum of $f$ (see Figure 1). Thus this is a limit case: for $\lambda_{3} \in$ $\left[2(2+\sqrt{6}) \lambda_{2}, \lambda_{2}\right]$ and $a \in I, f(a) \geq 0$. In fact, it is positive for $\lambda_{3} \in\left(2(2+\sqrt{6}) \lambda_{2}, \lambda_{2}\right]$. At the limit case there appear a zero at the endpoint $\sqrt{-\lambda_{3}}$ and the double zero $a_{z}$ in the admissible interval $I$. All $a \in I$ yield admissible solution of the IEP for EDMs.

For $\lambda_{3}<2(2+\sqrt{6}) \lambda_{2}$ the double zero turns into two zeros $z_{1}$ and $z_{2}$, and there is another zero $z_{3}<\sqrt{-\lambda_{3}}$ in $I$. The function $f$ is nonnegative in $\left[\sqrt{-\lambda_{2}}, z_{1}\right] \cup\left[z_{2}, z_{3}\right]$ and solutions of the IEP for EDMs exist only for parameters $a$ in this set.

Let us summarise the results.

Theorem 2.2. Let $I:=\left[\sqrt{-\lambda_{2}}, \sqrt{-\lambda_{3}}\right]$ and let the function $f$ be defined by (2.5). If:

(1) $\lambda_{2} \geq \lambda_{3} \geq 2(2+\sqrt{6}) \lambda_{2}$ and $a \in I$; or

(2) $\lambda_{3}<2(2+\sqrt{6}) \lambda_{2}$ and $a \in\left[\sqrt{-\lambda_{2}}, z_{1}\right] \cup\left[z_{2}, z_{3}\right]$, where $z_{1}, z_{2}, z_{3}$ are the zeros of the function $f$ in $I$,

then the matrix $D$ is an EDM.

The admissible region for the parameter $a$ is large, since it covers all possibilities, and thus the same triangle is described three times (for $a$ equal to each of the lengths of the triangle edges). In order to give a more precise result, let us consider some properties of the function $f$. 
Lemma 2.3. A local maximum of $f$ in $I$ ( for $\left.\lambda_{3}<2(2+\sqrt{6}) \lambda_{2}\right)$ is reached at

$$
\ell_{M}=\frac{1}{\sqrt[3]{6}} \sqrt{\frac{\sqrt[3]{6}\left(\lambda_{2}^{2}+\lambda_{2} \lambda_{3}+\lambda_{3}^{2}\right)}{Z\left(\lambda_{2}, \lambda_{3}\right)}+Z\left(\lambda_{2}, \lambda_{3}\right)}
$$

with

$$
Z\left(\lambda_{2}, \lambda_{3}\right):=\sqrt[3]{-9 \lambda_{2} \lambda_{3}\left(\lambda_{2}+\lambda_{3}\right)+W\left(\lambda_{2}, \lambda_{3}\right)}
$$

and

$$
W\left(\lambda_{2}, \lambda_{3}\right):=\sqrt{3} \sqrt{-\left(\lambda_{2}^{2}-2 \lambda_{2} \lambda_{3}-2 \lambda_{3}^{2}\right)\left(2 \lambda_{2}^{2}+2 \lambda_{2} \lambda_{3}-\lambda_{3}^{2}\right)\left(\lambda_{2}^{2}+4 \lambda_{2} \lambda_{3}+\lambda_{3}^{2}\right)} .
$$

Its local minimum in I is obtained at

$$
\ell_{m}=\sqrt{-\frac{\lambda_{2}}{6 \sqrt{2}}\left(\sqrt{\frac{k}{\sqrt[3]{r}}}-\sqrt{-\sqrt[3]{r}+\frac{54 \sqrt{2} t(1+t)}{\sqrt{\frac{k}{\sqrt[3]{r}}}+20\left(1+t+t^{2}\right)-\frac{49\left(1+t+t^{2}\right)^{2}}{\sqrt[3]{r}}}}\right)}
$$

with $t=\lambda_{3} / \lambda_{2}$,

$$
\begin{aligned}
& s=\sqrt{578+1734 t+4197 t^{2}+5504 t^{3}+4197 t^{4}+1734 t^{5}+578 t^{6}}, \\
& p=235+705 t+2139 t^{2}+3103 t^{3}+2139 t^{4}+705 t^{5}+235 t^{6}, \\
& r=p+\mathrm{i} 3 \sqrt{3} s\left(-2-3 t+3 t^{2}+2 t^{3}\right) \\
& k=\sqrt[3]{r^{2}}+10 \sqrt[3]{r}\left(1+t+t^{2}\right)+49\left(1+t+t^{2}\right)^{2} .
\end{aligned}
$$

Lemma 2.4. The function $f$ reaches its local maximum (2.6) in I at parameter a for which generating points form a perpendicular triangle (with perpendicular angle at $(b, c))$.

Proof. The generating points form a perpendicular angle at $(b, c)$ if and only if $d_{12}=d_{13}+d_{23}$ for the squared distances in (2.3). This simplifies into the relation

$$
a^{3}=\sqrt{-\left(a^{2}-\lambda_{1}\right)\left(a^{2}-\lambda_{2}\right)\left(a^{2}-\lambda_{3}\right)}
$$

Derivation of $f$ leads to

$$
\begin{aligned}
f^{\prime}(a)=2 a & \left(3 a \sqrt{-\left(a^{2}-\lambda_{1}\right)\left(a^{2}-\lambda_{2}\right)\left(a^{2}-\lambda_{3}\right)}\right. \\
& \left.-\frac{a^{3}\left(3 a^{4}+\lambda_{2} \lambda_{3}+\lambda_{1} \lambda_{3}+\lambda_{1} \lambda_{2}\right)}{\sqrt{-\left(a^{2}-\lambda_{1}\right)\left(a^{2}-\lambda_{2}\right)\left(a^{2}-\lambda_{3}\right)}}-\left(\lambda_{2}^{2}+\lambda_{2} \lambda_{3}+\lambda_{3}^{2}\right)\right) .
\end{aligned}
$$

By using the relation (2.7), the derivative vanishes. A solution of the equation $f^{\prime}(a)=0$ gives the local maximum (2.6). 
LEMMA 2.5. The generating points form an isosceles triangle for

$$
a=\sqrt{-\lambda_{2}}, \quad b=\frac{a}{2}=\frac{1}{2} \sqrt{-\lambda_{2}}, \quad c=\frac{1}{2} \sqrt{\lambda_{2}+2 \sqrt{2} \sqrt{\lambda_{3}\left(\lambda_{2}+\lambda_{3}\right)}} .
$$

The lengths of the triangle edges are

$$
\sqrt{-\lambda_{2}}, \quad \sqrt[4]{\frac{\lambda_{3}\left(\lambda_{2}+\lambda_{3}\right)}{2}}, \quad \sqrt[4]{\frac{\lambda_{3}\left(\lambda_{2}+\lambda_{3}\right)}{2}},
$$

and this gives the solution of the IEP for the EDM in (1.1).

PROOF. In order for the triangle to be isosceles, the following relation needs to be satisfied

$$
\sqrt{b^{2}+c^{2}}=\sqrt{(b-a)^{2}+c^{2}} .
$$

This yields $b=a / 2$. By using (2.2), it can be seen that the only admissible solution is $a=\sqrt{-\lambda_{2}}$. Computation of $c$ and the triangle edges lengths concludes the proof.

Recall that if $a$ is an admissible solution, then the choices $a=\sqrt{b^{2}+c^{2}}$ and $a=\sqrt{(b-a)^{2}+c^{2}}$ also give admissible solutions. It is enough just to study the case $a \in\left[\sqrt{-\lambda_{2}}, z_{1}\right]$, since the other two solutions lie in $\left[z_{2}, z_{3}\right]$.

Since $f\left(z_{1}\right)=f\left(z_{2}\right)=f\left(z_{3}\right)=0$, the corresponding solutions give collinear points $(0,0),(b, 0),(a, 0)$, where $a=z_{i}, b=b(a)=b\left(z_{i}\right), i=1,2,3$ (see (2.2)). The triangle edge lengths $a, \sqrt{b^{2}+c^{2}}$ and $\sqrt{(b-a)^{2}+c^{2}}$ are continuous functions of $a$ in $I$. Thus the shape of triangle changes from the degenerate triangle (the case $f\left(z_{1}\right)=f\left(z_{2}\right)=$ $f\left(z_{3}\right)=0$ ) up to the isosceles triangle (Lemma 2.5) when $a$ goes from $z_{1}$ down to $\sqrt{-\lambda_{2}}$. (The other two edge lengths converge from $z_{2}$ and $z_{3}$ into $\sqrt[4]{\lambda_{3}\left(\lambda_{2}+\lambda_{3}\right) / 2}$.)

We can now give a precise version of Theorem 2.2.

Theorem 2.6. Let $I:=\left[\sqrt{-\lambda_{2}}, \sqrt{-\lambda_{3}}\right]$ and let the function $f$ be defined by (2.5). If

(1) $\lambda_{2} \geq \lambda_{3} \geq 2(2+\sqrt{6}) \lambda_{2}$ and $a \in I$, or

(2) $\lambda_{3}<2(2+\sqrt{6}) \lambda_{2}$ and $a \in\left[\sqrt{-\lambda_{2}}, z_{1}\right]$, where $z_{1}$ is the first zero of the function $f$ in $I$,

then the matrix D is an EDM. This covers all possible distinct geometric configurations of generating points.

Note that for $\lambda_{3}=\lambda_{2}$ from (2.4) it follows that there is only one admissible solution $a=\sqrt{-\lambda_{2}}$.

Zeros of $f$ are continuous functions of $\lambda_{2}$ and $\lambda_{3}$. We can give good bounds on the zero $z_{1}$, and show that the admissible interval for $a$ tends to $\left\{\sqrt{-\lambda_{2}}\right\}$ for $\lambda_{2}$ and $\lambda_{3}$ far apart. 
Theorem 2.7. Let $t:=\lambda_{3} / \lambda_{2}$. Good lower and upper bounds for $z_{1}\left(a_{1}<z_{1}<a_{2}\right.$ and $\left.f\left(a_{1}\right)>0, f\left(a_{2}\right)<0\right)$ are

$$
a_{1}=\frac{t-\sqrt{2}}{t-2 \sqrt{2}} \sqrt{-\lambda_{2}}, \quad a_{2}=\frac{5 t-11}{5 t-22} \sqrt{-\lambda_{2}}, \quad t \geq 2(2+\sqrt{6}) .
$$

As $t \rightarrow \infty$, the admissible interval $\left[\sqrt{-\lambda_{2}}, z_{1}\right]$ shrinks to $\left\{\sqrt{-\lambda_{2}}\right\}$.

Proof. The upper and lower bounds were obtained as

$$
a_{1}=\frac{1}{2-\frac{1}{1-\frac{\sqrt{2}}{t}}}, \quad a_{2}=\frac{1}{2-\frac{1}{1-\frac{22}{10 t}}} .
$$

As $t \rightarrow \infty$, both $a_{1}$ and $a_{2}$ converge to $\sqrt{-\lambda_{2}}$. Furthermore,

$$
a_{2}-a_{1}=\frac{\sqrt{5}(5 \sqrt{2}-11) t}{(t-2 \sqrt{2})(5 t-22)} \sqrt{-\lambda_{2}}
$$

tends to 0 for large $t$. Note that for $2(2+\sqrt{6}) \doteq 8.89898 \leq t<8.89907, f\left(a_{2}\right)>0$, so here $a_{2}$ does not give an upper bound for $z_{1}$. A better upper bound in this case can be obtained, but then it is not as good for larger $t$.

\section{Inverse eigenvalue problem and bordered matrices}

First let us give a relation between eigenvalues of a symmetric hollow matrix and its bordered matrix.

Theorem 3.1. Let $\lambda_{1}, \lambda_{2}, \ldots, \lambda_{n+1}$ be given real numbers with $\sum_{i} \lambda_{i}=0$. Let $M \in \mathbb{R}^{n \times n}$ be a symmetric hollow matrix, and let $M=U D U^{T}$ be its eigendecomposition with $D=\operatorname{diag}\left(d_{1}, \ldots, d_{n}\right), U^{T} U=I$. Let

$$
a_{i}:=\sqrt{-\frac{\prod_{j=1}^{n+1}\left(d_{i}-\lambda_{j}\right)}{\prod_{j=1 \neq i \neq i}^{n}\left(d_{i}-d_{j}\right)}}, \quad i=1,2, \ldots, n,
$$

and $\boldsymbol{b}:=U \boldsymbol{a}$. Then the matrices

$$
\left[\begin{array}{ll}
M & \boldsymbol{b} \\
\boldsymbol{b}^{T} & 0
\end{array}\right] \text { and }\left[\begin{array}{cc}
D & \boldsymbol{a} \\
\boldsymbol{a}^{T} & 0
\end{array}\right]
$$

are similar and have eigenvalues $\lambda_{1}, \lambda_{2}, \ldots, \lambda_{n+1}$.

Proof. Since

$$
\left[\begin{array}{cc}
U^{T} & 0 \\
0 & 1
\end{array}\right]\left[\begin{array}{cc}
M & \boldsymbol{b} \\
\boldsymbol{b}^{T} & 0
\end{array}\right]\left[\begin{array}{cc}
U & 0 \\
0 & 1
\end{array}\right]=\left[\begin{array}{cc}
D & \boldsymbol{a} \\
\boldsymbol{a}^{T} & 0
\end{array}\right]
$$


the matrices are orthogonally similar. The right-hand-side matrix is an arrow matrix. Its characteristic polynomial is (see, for example, [7])

$$
f(\lambda)=\left(\lambda-\lambda_{1}\right)\left(\lambda-\lambda_{2}\right) \cdots\left(\lambda-\lambda_{n+1}\right)=\lambda+\sum_{k=1}^{n} \frac{a_{k}^{2}}{d_{k}-\lambda} .
$$

A simplification of the right-hand side and comparison of coefficients of the polynomials obtained gives a polynomial system that needs to be studied. Its solution is $(3.1)$.

Corollary 3.2. Let the assumptions of Theorem 3.1 be satisfied. Let $d_{i}=\lambda_{i+1}$, $i=2,3, \ldots, n$ in (3.1). Then $\boldsymbol{a}=\beta \boldsymbol{e}_{1}, \beta>0$ and $\boldsymbol{b}=\beta \boldsymbol{u}_{1}$. This gives Fiedler's construction of a larger EDM from a given one by using Perron's theorem and properties of a specific bordered matrix (see [5]).

In order to construct an EDM with given eigenvalues $\lambda_{1} \geq 0 \geq \lambda_{2} \geq \cdots \geq \lambda_{n+1}$ with $\sum_{i} \lambda_{i}=0$, we need to find $d_{1}, d_{2}, \ldots, d_{n}$ such that $\lambda_{i+1} \leq d_{i} \leq \lambda_{i}, i=1,2, \ldots, n$, and that the matrix obtained is an EDM. The first relation gives us a lot of freedom, but the second one is a hard nut to crack.

Assume that the matrices $M \in \mathbb{R}^{n \times n}$ and

$$
C:=\left[\begin{array}{cc}
M & b \\
\boldsymbol{b}^{T} & 0
\end{array}\right] \in \mathbb{R}^{(n+1) \times(n+1)}
$$

are nonsingular. The eigendecomposition $M=U D U^{T}$, with $U^{T} U=I, \quad D=$ $\operatorname{diag}\left(d_{1}, d_{2}, \ldots, d_{n}\right)$ and $\boldsymbol{b}=U \boldsymbol{a}$, together with

$$
\left[\begin{array}{cc}
D & \boldsymbol{a} \\
\boldsymbol{a}^{T} & 0
\end{array}\right]^{-1}=\left[\begin{array}{cc}
D^{-1} & 0 \\
0 & 0
\end{array}\right]-\frac{1}{\boldsymbol{a}^{T} D^{-1} \boldsymbol{a}}\left[\begin{array}{c}
D^{-1} \boldsymbol{a} \\
-1
\end{array}\right]\left[\boldsymbol{a}^{T} D^{-1}-1\right],
$$

yield the solution

$$
\begin{aligned}
& \boldsymbol{w}=U D^{-1} U^{T} \boldsymbol{e}-\frac{1}{\boldsymbol{a}^{T} D^{-1} \boldsymbol{a}} U D^{-1} \boldsymbol{a} \boldsymbol{a}^{T} D^{-1} U^{T} \boldsymbol{e}+\frac{1}{\boldsymbol{a}^{T} D^{-1} \boldsymbol{a}} U D^{-1} \boldsymbol{a}, \\
& \alpha=\frac{1}{\boldsymbol{a}^{T} D^{-1} \boldsymbol{a}}\left(\boldsymbol{a}^{T} D^{-1} U^{T} \boldsymbol{e}-1\right)
\end{aligned}
$$

of the system

$$
\left[\begin{array}{ll}
M & b \\
\boldsymbol{b}^{T} & 0
\end{array}\right]\left[\begin{array}{l}
\boldsymbol{w} \\
\alpha
\end{array}\right]=\left[\begin{array}{l}
\boldsymbol{e} \\
1
\end{array}\right]
$$

The matrix $C$ is an EDM if and only if

$$
\left\langle\left[\begin{array}{l}
\boldsymbol{w} \\
\alpha
\end{array}\right],\left[\begin{array}{l}
\boldsymbol{e} \\
1
\end{array}\right]\right\rangle \geq 0
$$


A short computation simplifies this relation to

$$
\boldsymbol{e}^{T} U D^{-1} U^{T} \boldsymbol{e}-\frac{1}{\boldsymbol{a}^{T} D^{-1} \boldsymbol{a}}\left(\boldsymbol{a}^{T} D^{-1} U^{T} \boldsymbol{e}-1\right)^{2} .
$$

Since $M^{-1}=U D^{-1} U^{T}$ and since the matrix $M$ is an EDM, there exists $\widetilde{w}$ such that $M \widetilde{\boldsymbol{w}}=\boldsymbol{e}, \widetilde{\boldsymbol{w}}^{T} \boldsymbol{e} \geq 0$. Thus the first term in (3.3) is nonnegative. The following lemma implies that the last one is nonnegative too.

Lemma 3.3. For D nonsingular,

$$
\boldsymbol{a}^{T} D^{-1} \boldsymbol{a}>0
$$

Proof. Let us define matrices

$$
A_{1}:=\left[\begin{array}{cc}
D^{-1} & 0 \\
\boldsymbol{a}^{T} D^{-1} & -1
\end{array}\right], \quad \widehat{D}:=\left[\begin{array}{cc}
D & \boldsymbol{a} \\
\boldsymbol{a}^{T} & 0
\end{array}\right], \quad A_{2}:=\left[\begin{array}{cc}
I & D^{-1} \boldsymbol{a} \\
0 & \boldsymbol{a}^{T} D^{-1} \boldsymbol{a}
\end{array}\right] .
$$

Clearly $A_{1} \widehat{D}=A_{2}$. Thus $\operatorname{det} A_{1} \operatorname{det} \widehat{D}=\operatorname{det} A_{2}$. Since $\operatorname{det} A_{1}=-\operatorname{det} D^{-1}$, $\operatorname{det} \widehat{D}=$ $\prod_{i=1}^{n+1} \lambda_{i}$ and $\operatorname{det} A_{2}=\boldsymbol{a}^{T} D^{-1} \boldsymbol{a}$,

$$
\boldsymbol{a}^{T} D^{-1} \boldsymbol{a}=-\frac{\prod_{i=1}^{n+1} \lambda_{i}}{\prod_{i=1}^{n} d_{i}}>0
$$

This concludes the proof.

As an example, let us consider the step from $n=2$ to $n=3$.

Lemma 3.4. Let $n=2$. There exists $d_{2}$ such that the second term in (3.3) vanishes, and $\boldsymbol{w}^{T} \boldsymbol{e}=\widetilde{\boldsymbol{w}}^{T} \boldsymbol{e}=-2 / d_{2}$. This solution does not give the maximal possible value $\boldsymbol{w}^{T} \boldsymbol{e}$.

Proof. For $n=2$,

$$
D=\left[\begin{array}{cc}
-d_{2} & 0 \\
0 & d_{2}
\end{array}\right], \quad U=\frac{1}{\sqrt{2}}\left[\begin{array}{cc}
1 & -1 \\
1 & 1
\end{array}\right]
$$

and

$$
\boldsymbol{a}=\left[\sqrt{-\frac{\left(d_{1}-\lambda_{1}\right)\left(d_{1}-\lambda_{2}\right)\left(d_{1}-\lambda_{3}\right)}{d_{1}-d_{2}}}, \sqrt{-\frac{\left(d_{2}-\lambda_{1}\right)\left(d_{2}-\lambda_{2}\right)\left(d_{2}-\lambda_{3}\right)}{d_{2}-d_{1}}}\right]^{T} .
$$

With $\boldsymbol{b}=U \boldsymbol{a}$ and $M=U D U^{T}$, we can construct the matrix $C$ in (3.2). If $C$ is nonsingular, we can apply the previous computation, and obtain

$$
\boldsymbol{w}^{T} \boldsymbol{e}=\frac{2 d_{2}^{2} \sqrt{-\frac{\left(d_{2}+\lambda_{1}\right)\left(d_{2}+\lambda_{2}\right)\left(d_{2}+\lambda_{3}\right)}{d_{2}}}-\lambda_{2} \lambda_{3}\left(\lambda_{2}+\lambda_{3}\right)+d_{2}\left(\lambda_{2}^{2}+\lambda_{2} \lambda_{3}+\lambda_{3}^{2}\right)}{d_{2} \lambda_{2} \lambda_{3}\left(\lambda_{2}+\lambda_{3}\right)} .
$$




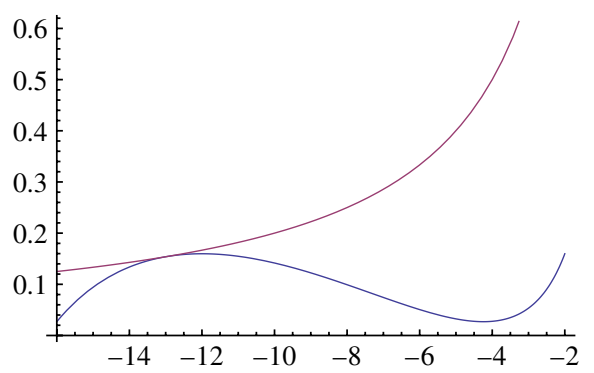

FIGURE 2. The value $d_{2}$ which gives unchanged $\boldsymbol{w}^{T} \boldsymbol{e}=-2 / d_{2}$ is obtained as the intersection of curves. Here $\lambda_{2}=-2, \lambda_{3}=-16$.

For $n=2, \widetilde{\boldsymbol{w}}^{T} \boldsymbol{e}=-2 / d_{2}$. The solution of the system $\boldsymbol{w}^{T} \boldsymbol{e}=-2 / d_{2}$ is

$$
d_{2}=-\lambda_{2} \frac{2 \sqrt[3]{6}\left(1+t+t^{2}\right)+\mathrm{i}(\mathrm{i}+\sqrt{3}) T^{2}}{2 \sqrt[3]{6} T} \in \mathbb{R}
$$

with

$$
T:=\sqrt[3]{9 t+9 t^{2}-\mathrm{i} \sqrt{3} \sqrt{2+6 t-15 t^{2}-40 t^{3}-15 t^{4}+6 t^{5}+2 t^{6}}} .
$$

The solution is obtained as the curve $-1 / d_{2}$ touches the curve $\boldsymbol{w}^{T} \boldsymbol{e}$ (see Figure 2). For data in Figure 2, the solution $d_{2}=-12.9694$ yields $\boldsymbol{w}^{T} \boldsymbol{e}=-2 / d_{2}=0.154209$. But the values $d_{2}=-12$ and $d_{2}=-2$ give a larger $\boldsymbol{w}^{T} \boldsymbol{e}=0.159722$.

The study of the general case is more complex. We need to find $d_{1}, d_{2}, \ldots, d_{n}$ such that $\lambda_{i+1} \leq d_{i} \leq \lambda_{i}, i=1,2, \ldots, n$, and that expression (3.3) is nonnegative. Furthermore, the matrix $M$ has to be hollow $\left(m_{i i}=0, i=1,2, \ldots, n\right)$ and an orthogonal matrix $U$ is required such that $M$ and $\boldsymbol{b}$ are nonnegative.

\section{References}

[1] R. Balaji and R. B. Bapat, 'Block distance matrices', Electron. J. Linear Algebra 16 (2007), 435-443.

[2] M. T. Chu, 'Constructing a Hermitian matrix from its diagonal entries and eigenvalues', SIAM J. Matrix Anal. Appl. 16 (1995), 207-217.

[3] M. T. Chu and G. H. Golub, Inverse Eigenvalue Problems: Theory, Algorithms, and Applications (Oxford University Press, Oxford, 2005).

[4] J. Dattorro, Convex Optimization and Euclidean Distance Geometry (Meboo, Palo Alto, CA, 2005).

[5] M. Fiedler, 'Eigenvalues of nonnegative symmetric matrices', Linear Algebra Appl. 9 (1974), 119-142.

[6] M. Fiedler, 'Some inverse problems for elliptic matrices with zero diagonal', Linear Algebra Appl. 332-334 (2001), 197-204.

[7] G. H. Golub and C. F. van Loan, Matrix Computations, 3rd edn (Johns Hopkins University Press, Baltimore, MD, 1996).

[8] T. L. Hayden, R. Reams and J. Wells, 'Methods for constructing distance matrices and the inverse eigenvalue problem', Linear Algebra Appl. 295 (1999), 97-112. 
[9] G. Jaklič and J. Modic, 'On properties of cell matrices', Appl. Math. Comput. 216 (2010), 2016-2023.

[10] T. J. Laffey and H. Šmigoc, 'Construction of nonnegative symmetric matrices with given spectrum', Linear Algebra Appl. 421 (2007), 97-109.

[11] I. J. Schoenberg, 'Metric spaces and positive definite functions', Trans. Amer. Math. Soc. 44 (1938), 522-536.

GAŠPER JAKLIČ, FMF and IMFM, University of Ljubljana, Jadranska 19, 1000 Ljubljana, Slovenia

and

IAM, University of Primorska, Jadranska 19, 1000 Ljubljana, Slovenia e-mail: gasper.jaklic@fmf.uni-lj.si

JOLANDA MODIC, FMF, University of Ljubljana and XLAB d.o.o., Pot za Brdom 100, 1000 Ljubljana, Slovenia

e-mail: jolanda.modic@gmail.com 